\title{
ENTRE LA COYUNTURA Y LA UTOPÍA. EL SUEÑO DE LO IMPOSIBLE
}

\author{
por ÍÑGo DIAZ CuEvas \\ Decano de la Facultad de Ciencias Veterinariasy Pecuarias \\ Universidad de Chile
}

\begin{abstract}
RESUMEN
Se hacen algunas reflexiones en tomo a la sensible dualidad de lo urgente o cotidiano enfrentado a lo importante o trascendente. Se discute esta proposición disyuntiva como una explicación al freno de la aproximación utópica, a situaciones universitarias consideradas como ideales para el desarrollo institucional. Se analiza la capacidad de poner en duda, con la debida eficiencia y equidad, los modelos y principios universitarios. Finalmente, se realiza una enumeración de aquellos temas que requieren tratamiento prioritario: relación Estado-Universidad, financiamiento a las Universidades estatales y estatuto y órgano superior de gobierno universitario.
\end{abstract}

Una de la realidades más intangibles y difíciles de estructurar es el logro de consenso frente a un modelo de Universidad. Es una especie de fuerza superior la que nos lleva a involucrarnos de tal modo en la vida universitaria, que nos impide la reflexión sobre lo que debe ser una Casa de Estudios Superiores. Cada situación o experiencia académica nueva que vivimos enseña una realidad que, a su vez, nos obliga a reformular más de una vivencia o principio que sentíamos sólido y que pensábamos consolidado.

Recuerdo con cierta nostalgia los análisis y reflexiones universitarios ocurridos en la bella década del '70, cuando dábamos los primeros pasos en la vida universitaria. Los jóvenes estudiantes de entonces nos sumergíamos en extensas y rigurosas elucubraciones, algunas de ellas ideológicas, en tomo al estudio de un modelo de Universidad. La necesidad de 
ese momento era, a no dudarlo, aferrarse a un esquema universitario que nos permitiera salir de esa estructura académica anquilosada y poco lubricada que nos había heredado el medio siglo. En algunas oportunidades discutíamos extensamente el concepto de "nueva Universidad y sociedad global". Decíamos que esta nueva Universidad debía responder a los desafíos de la sociedad, democratizandosu acceso y sus estructuras, garantizando el ingreso al máximo de postulantes, haciendo la selección por la vía natural, en un marco de jerarquía de méritos. Rechazábamos el concepto de institución elitista con el argumento de que muchos no llegarían a la Educación Superior más por las limitaciones de su condición económica, que por las de sus aptitudes académicas. Estábamos convencidos de que la sociedad encontraría en la comunidad universitaria un modelo referencial. Priorizábamos de forma cardinal la autonomía frente a poderes extraños, lo que nos permitiría frenar cualquier ataque a ésta. Decíamos que la ciencia no admitía ningún techo y que podía crecer sin límites y que la Universidad debía darse cuenta de que en su interior vivían entidades con ser propio, que tenían que ser garantizadasy defendidas como cosas sagradas.

Esta Universidad asumía finalmente su papel dinámico para orientar los cambios sociales. Siguiendo a Scherz, establecíamos que "el afán de la Universidad de constituirse en un macrocosmos idealizado y referencial con respecto a la sociedad global, encuentra a través del modelo expuesto una de sus posibles expresiones. En su seno se configura el ámbito favorable para la formación del microcosmos humano"2.

A treinta años de distancia, son evidentes los matices y connotaciones que se le puedan dar a estas concepciones que movieron y remecieron la vida universitaria y nacional.

Tiendo a pensar hoy día que la cuestión central de discusión, como hace tres décadas, es la capacidad de poner en duda los modelos $\mathbf{y}$ principios universitarios, corriendo el riesgo de caere $n$ planos de confusa relatividad o en esquemas rígidos que no permitan salidas reales a los temas y desafios universitarios. El espacio de tolerancia para el enfoque de los temas de grandeza es hoy día estrecho, lo que conlleva actitudes peligrosamente dicotómicas. Por una parte, retornamos con avidez a nuestros espacios más conocidos para rescatar ancestrales principios universitarios, para repensar aquellos valores inamovibles y reconocidos por todos o, por el contrario, optamos por proyectamos hacia una

${ }^{1}$ Scherz, Luis, El camino de la revolución universitaria, Editorial del Pacífico, Santiago de Chile, 1968.

${ }^{2}$ Scherz, Luis, op. cit. 
modemidad genérica, ubicando en niveles de privilegioa los indicadores de eficiencia de la función académica, como único elemento de reaiidad institucionalfrente a nuestras audiencias o clientes.

Recordandoa Ortega y Gasset cuando expresaba "hacercambiosen la Universidad es como remover cementerios", debemos reconocer lo dificultoso que resulta la innovación en la Educación Superior. Sin embargo, necesitaremos combinar adecuadamente la reflexión con la prudencia para realizar los cambiosque se requieren, sin por ello vulnerar principios o minimizar riesgos.

Cuestiones tan trascendentes como encontrar los caminos para definir la misión institucional de la Universidad de Chile no tienen espacio por la urgencia de resolver, por ejemplo, el problema cotidiano de la docencia de pregrado o el de la restringida agilidad de la función administrativa de apoyo que disponemos. Situaciones de la categoría de la decisión frente a la evaluación institucionaly su compromiso con planes estratégicos, se contrastan, a menudo, con la necesidad de preocuparse de presupuestos deficitarios para asumir los gastos de mantención o funcionamiento que requiere la Corporación. Hoy díi la discusión sobre las relaciones entre el Estado y la Universidad, con toda su riqueza valórica, es reducida, y en definitiva, confundida con el tema de la entrega de recursos; $y$, por cierto, rodeada de opiniones interesantes pero también de corto plazo y con notorios intereses particulares.

Cómo no pensar que la docencia de pregrado o la administración son temas importantes; cómo soslayar el hecho que los problemas relacionados con el gasto corriente de la Universidad consumen parte importante de nuestro quehacer cotidiano, o que las acciones que es necesario ejecutar para enfrentar las restricciones económicas nos demandan más tiempo que el razonable en nuestro vivir académico.

Pese a las rigideces estructurales, valóricas o temporales que enfrentamos, debemos preguntarnos hoy díi: ¿Tiene sentido pensar acerca del sentido de la Universidad? ¿Podemos establecer espacios de tolerancia para dialogar y reflexionar sobre la misión universitaria, o sobre el tema, por cierto novedoso, de la evaluación institucional,o el de la relación del Estado y la sociedad, o de las nuevas formas de gobierno universitarioy estructura académica, o el de la necesidad de gestionar adecuadamente la Universidad, ofortalecer la actividad creativa e incitar a ella, o imaginar los mecanismos de reconocimiento que pudiesen alcanzar aun a las relaciones interpersonales, o por último, poder pensar casi sin limitaciones el tema del espacio geográfico y la inserción urbana de la Universidad? 
Así como los espacios de tolerancia son estrechos, con frecuencia tendemos a reducir los problemas a disyuntivas falsas. Con facilidad polarizamos los términos de la discusión, lo que nos lleva, por ejemplo, a plantear la calidad académica de una función universitaria como opuesta a una situación económica sana. Con frecuencia nos sorprendemos en la convicción que la función académica no podrá realizarse jamás con presupuestos acotados y finitos. Algunas veces nos encontramos en dilemas que enfrentan los temas relativos a la animación cultural, como contrapuestos a una administración eficiente de aquella actividad universitaria. También incurrimos en la creencia de que lo que traspasa esos "muros de piedra y de sol"no es evaluable, forma parte del patrimonio intangible de la Universidad y, en consecuencia, no puede someterse a juicio.

Creo que podemos estar acercándonos al centro de estas reflexiones: al escenario en que la coyuntura y sus urgencias no nos permiten preocuparnos de lo importante, vale decir, de las utopías que cada universitario tiene la obligación de vocear con todas sus fuerzas. ¿Qué otra cosa es la Universidad sino una secuencia de utopías que se transformarán, de acuerdo a las condicionantes ambientales, en planes, proyectos o sistemas amables, algunas veces realizables?¿Qué otra cosa es la Universidad sino hacerse del tiempo, más allá de las urgencias demandantes y sofocantes, para poder asombrarse con ideas nuevas (en algún momento decíamos revolucionarias), sobre temas que necesitan un ámbito de reflexión y tranquilidad para generar las fuerzas dinámicas del movimiento y desarrollo innovador de la institución?¿Qué situación más cercana a "la comunidad de maestros y discípulos" que poder tener la claridad de conceptos y la magnificencia intelectual para adentrarse tanto en los temas urgentes como en la reflexión de lo importantey, en consecuencia, de lo permanente y trascendente?:Por qué el escenario de las cuestiones coyunturales, en interacción con los grandes temas de relevancia será el sueño de lo imposible?

Me resisto a aceptar que no puedan aproximarse ecuaciones que permitan resolver con eficiencia y equidad esta proposición disyuntiva. Pese a que haya encabezado estas reflexiones con ese título, me reservo como tesoro entrañable la posibilidad y, aún más, el derecho de poder siempre soñar con ciertas utopías, abrir espacios para el asombro, creer en la capacidad de abordar el presente con sus requerimientoscomentes y el futuro con sus grandezas casi irrealizables. Me guardo con gran fervor el pensar que es posible y necesario para este hoy día, realizar lo imposible. 
Si los anteriores planteamientos tienen algún atisbo de realidad, intent a encontrar, o más bren, ordenar alguna explicación a la cuestión de lo urgente y de to trascendente. Creo que un camino es buscar en las vivencias universitarias las variables que la puedan esclarecer.

En estos términos, la urgencia ha sido el elemento común que ha ordenado la vida universitaria en los Últimos treinta años. Pese a no compartir sentencias dramáticas tales como "laUniversidad atraviesa por una profunda chis", debo estar de acuerdo que desde los inicios de la Reforma Universitaria en mayo de 1968, nuestra Universidad de Chile ha vivido procesos profundos de cambio y reestructuraciones que no han podido finalizar con una adecuada solución de continuidad ni menos con los necesarios procesos de maduración y evaluación. De hecho, el proceso de Reforma Universitaria iniciado con la fuerza avasalladora marcada por el impetu estudiantil, entró por caminos que de una u otra manera fueron desvirtuados por las urgencias externas, por cierto respetables 0 , al menos, explicables. Ese proceso fue discontinuado por una suerte de "urgencia de fuerza mayor", entrando la Universidad de Chile a vivir el régimen dictatorial en las formas de una administración universitaria delegada o de intervención militar. Vivimos durante esos años una de las formas más abyectas de urgencia, cual era la de recibir las ordenanzas de autoridades administrativasinstaladas, las que a su vez debían concretar medidas de apariencia universitaria. Durante todo ese período, la actividad académica institucional y, por cierto, la de cada uno de los profesores, estuvo marcada por la urgencia de sobreviviren lo desconocido, más allá de las grotescas situacionesque padeció casi toda la Universidad.

Un matiz de esa misma urgencia se empezó a vivir cuando hacia mediados de la década del ' 80 aparecieron los primeros signos de reacción académica autónoma. A no dudarlo, era más urgente terminar con la intervención militar en la Universidad que detenerse a observar las tendencias por las que se encaminaba la Educación Superior en la mayoría de los países del mundo. Sus nuevas formas de administración o las crecientes reducciones del financiamiento estatal obligaban a diseñar muy audaces estrategias de desarrollo. En esos años no hubo tiempo ni espacio para damos cuenta de que, aun retornando a la Democracia y por consiguiente, terminada la intervención universitaria, sería extraordinariamente difícil revertir la orientación iniciada en la década de los '70y generalizada en los ' 80 , en relación al cuestionamiento de la idea del Estado Benefactor $\mathbf{y}$, por tanto, la obligación de diseñar escenarios que permitieran estimar con alguna certeza los efectos que podía tener esta retracción de las responsabilidades del Estado. 
Ciertamente, cuando terminó la intervención, la urgencia de la Universidad fue establecer procedimientos operativos que nos permitieran disponer de una gestión académica sostenida plenamente por los pares. La urgencia de disponer de un gobierno académico con espaciopara sentirnos haciendo Universidad nos impidió detenemos por algún momento a ordenar y priorizar nuestra agenda.

Así, los primeros años de gobierno académico han sido escasos para salir de las urgencias con las que convivimostantos años: federalismo en lo estructural, ausencia valórica del bien común, complicaciones para visualizar las misiones sustantivas de la institución, dificultades para iniciar formas de gobierno eficiente y representativoo serias inercias para imaginar nuevos diseños en las acciones académicas. Las urgencias nos habían enseñado que la institución no era el tema de debate y que el individualismo se transformaba en un adecuado escenario de vida. Lo anterior, a mi juicio, explica parcialmente la imposibilidad de emprender iniciativas ambiciosas y en algunos casos riesgosas, debido a que tenemos urgentes problemas en el quehacer académico, que deben ser tratados con prioridad y celeridad.

Por ahora no quiero buscar otras explicaciones, que ciertamente existen, sino avanzar en algunos temas de trascendencia que considero no pueden postergarse en función de las urgencias del presente.

Un aspecto de trascendental importancia y que debido a los acontecimientos nacionales por todos conocidos se ha postergado más allá de lo prudente, es el tema de las relaciones entre el Estado y la Universidad de Chile, lo que impulsa a pensar en la conveniencia, de orden nacional, de trabajar con más dedicación en las relaciones entre el Estado y el sistema de Educación Superior. Todo lo anterior ha significadoque las instituciones universitarias han debido abordar serios inconvenientes debido a su gran diversidad, en un sistema demasiado abierto. Esto, sin un adecuado cuerpo de normas regulatorias, hace que la calidad del servicio académico que se entrega sea puesta en tela de juicio cada vez que se enfrenta alguna urgencia. No cabe duda que la diversificación del sistema de Educación Superior es por sí sola una muy ventajosa característica que apunta a la solución educacional del país. Pero, al mismo tiempo, esta diversificación, sin el necesario correiato en cuanto a medidas de regulación, provoca serias inestabilidades en el sistema y, con frecuencia, expone a las instituciones a situacionesde fuerte inequidad.

Cualquierenfoque analítico del sistema de Educación Superior chileno hace resaltar diferencias muy marcadas en cuanto al tipo de instituciones 
Universidades junto a Institutos Profesionales,y Centros de Formación Técnica - al tamaño que presentan (de gran complejidad, medianas y pequeñas), a la orientación temática o cobertura disciplinaria (restringidas o específicas y completas), al tipo de misiones o funciones que realizan, a la propiedad de la institucióny a los sistemas de financiamiento. No cabe duda que estas características, sin una regulación adecuada, producen serios inconvenientes en relación a la calidad académica, o cuando menos generan incógnitas de credibilidad o fe pública.

Además de la diversificación del sistema, continúan manteniéndose algunas características preocupantes en relación a la población estudiantil. Pese al aumento de matrícula observado, siguen siendo muy pocos los estudiantes que acceden a la Educación Superior y, por sobre todo, aumenta la presencia de los estratos con mayor capacidad económica, haciendo perverso el círculo en relación a la capacidad intelectual y capacidad económica, A su vez, la oferta educativa dice más relación con los márgenes económicos de las carreras que con las necesidades nacionales o con las demandas de nuestros jóvenes. Lo anterior hace que exista una gran cantidad de vacantes no utilizadas, lo que aporta ctro signo surrealista al tema.

El tema diversidad-regulaciónno sólo debe entenderse frente al emergente sector privado de la Educación Superior, sino también al grupo de instituciones que reciben aporte fiscal o que pertenecen al Estadb. De alguna forma, el devenir del sistema ha transitadopor sendas que no han tenido el necesario escrutinio del Estado y que ha provocado en algunas áreas desarrollos virtuales y efectos directos e indirectos negativos. $\mathrm{El}$ tema es de tal trascendencia que nuestra Universidad lo abordó en presencia del Presidente de la República, durante la inauguración del año académico en 19943. En esa oportunidad, el Rector indicaba: "No hay duda que en los últimos $\mathbf{2 0}$ años han estado ocurriendo hechos y se han tomado decisiones respecto al sistema universitario que, por cierto, se relacionan con un determinado modelo de funcionamientoeconómicoy social, pero lo hacen sólo superficialmente, pues ha sido el resultado intuitivo y algo mecánico de la aplicación de algunos conceptos de carácter económico financiero, trasladados acríticamente al mundo del conocimientoy la cultura y, con ello, al sistema universitario.

"Enuna palabra, el Estado chileno, en esta particular situación histórica, no ha generado políticas explícitas respecto a la Universidad y su

${ }^{3}$ Lavados, Jaime, La Universidad y el Estado. Discurso del Rector de la Universidad de Chile en la inauguración del año académico, Editorial Universitaria, Santiago de Chile. 1994. 
papel en el desarrollo moderno, que correspondan a ideas fuerza, de algún modo, consensuadas entre diversos actores (Gobierno, Parlamento, clase política, medios de comunicación, universitarios intelectuales, empresarios). Creo que se trata de uno de los aspectos más vitales y menos aclarados de los que se ha dado en llamar el nuevo rol del Estado.

"Llamala atención la poca relevancia que ha tenido, en estas discusiones, un análisis del nuevo papel del Estado en la creación, difusión y uso de conocimiento, y en el fortalecimiento y desarrollo de la cultura. Porque, en definitiva, de lo que se trata es de examinar de qué manera, con qué mecanismos, o con cuáles instrumentos el país avanza, como necesita, en el mejoramiento de su Educación Superior, en la maduración de su capacidad cientifica y tecnológica y en el estímulo a su perfeccionamiento cultural".

Terminaba el Rector indicando: "Asímiradas las cosas, hay mucho por hacer, y hay que hacerlo pronto. Muchas modernizaciones y cambios al interior de nuestras instituciones, y varias decisiones de política de Estado que no pueden esperar. No podemos continuar como observadores indiferentes de un futuro en riesgo. Tenemos, debemos asumir las tareas que a cada cual corresponda. No sólo el Gobierno y el Parlamento sino, también, la comunidad universitaria y, junto a ellos, los medios de comunicación y la clase política, los empresarios y los intelectuales. Es decir, todo Chile, porque se trata de una empresa que a toda la nación importa"4.

Lo anterior nos lleva a pensar que aparece con fuerza la necesidad de disponer de definicionesexplícitasen el ámbitode la Educación Superior. El desarrollo del sistema debe ser producto de las medidas adoptadas a la luz de visiones de conjunto y de análisis amplios y no por decisiones parciales, tomadas en atmósferas sesgadas e interesadas, y en definitiva, con efectos de certera ineficiencia. En estas defmiciones de política, un elemento primordial es la relación entre el Estado y las Universidades estatales.

La primera condición que habría que establecer es la categoría de estatal de una Universidad, la que hasta hoy día está definida sólo por la propiedad. $\mathrm{Si}$, además de lo anterior, buscamos otras característicasque definan esta condición, con pesadumbre nos daremos cuenta que es muy difícil encontrarlas. Veamos: Si se analiza el monto de recursos que éstas logran del Estado, observamos de hecho que existen Universidades privadas que reciben mayor aporte que algunas estatales. Las funciones 
y objetivos que eventualmente las pudiesen diferenciar, tales como el postgrado en ateas de alta especificidad, investigación científica y tecnológica de punta, funciones de servicio público o actividades de animación cultural, no son incentivadas con los estímulos adecuados, y por tanto, tienden a perder identidad. En resumen, hoy más que antes se requiere un esfuerzo conjunto para explorar la misión institucional de la Universidad estatal, de acuerdo con los intereses y necesidades nacionales.

Con una fuerte intencionalidad he dejado un tanto al margen el tema del financiamiento universitario, en especial, el de las Universidades estatales: Como es sabido, en los últimos veinte años se ha venido observando un descenso importante del financiamiento fiscal de la Educación Superior. Las cifras indicaban que en los inicios de la década del '70,el gasto fiscal en Educación Superior bordeaba el 1,7\% del PGB, valor que para 1990 no alcanzaba el $0,5 \%$, tendencia que se revierte de forma marginal para llegar al 0,6\% en 1992. El tema de las caídas en los aportes del Estado no nos debiera preocupar por su tendencia -generalizada en numerosos paises - simo por los niveles que ha alcanzado. Aun considerando que es bueno para una Universidad del Estado lograr recursos diferentes a los de origen fiscal, no es equitativo ni saludable que el Estado financie a sus Universidades con los montos que observamos hoy día. Sin riesgo a equivocarme, creo que no existe experiencia en el mundo en que el Fisco aporte a sus instituciones cifras del orden del 25-30\% del presupuesto universitarioy que, al mismo tiempo, las considere estatales. Creo que en este punto la necesidad de encuentro con el Estado es fundamental, bajo la necesidad de consensuar fundamentos, criterios y mecanismos de financiamientoque permitan a nuestras Universidades continuar haciendo aportes importantes al desarrollode país.

Dos aspectos que me gustaría poder insinuar y que, a no dudarlo, se muestran como de elevada importancia, son los temas del estatutoy del órgano superior de gobierno de la Universidad, y el de las formas de gestión universitaria. Estos son precisamenteproblemas que cada institución debe abordar, que permiten expresar identidady realidad institucional, pero que al mismo tiempo son reflejode las condicionesambientales del sistemà.

Al abordar el tema del Estatuto, se aprecia que el actual Decreto con Fuerza de Ley № 153, del Ministerio de Educación, Estatuto de la Universidad de Chile, describe con detalle el tema del gobiernoy la estructura, además de tocar tangenciaimente aspectos relativos a los académicos y estudiantes y a la organización de los estudios. Llama la atención que no 
se establezca nada en relación a los propósitos permanentes de la institución y sus características de relación con el Estado, salvo alguna declai xción de intenciones (el Presidente de la República es el patrono de la Universidad de Chile). Sin lugar a dudas, el incorporar cuestiones de estructura organizativa y, por lo tanto, susceptibles a modificaciones temporales, introduce infiexibilidades relativas justamente a temas de estructuray de gestión o en las tareas de planificación. A la larga, cuando se incorpora la estructura organizativa a un estatuto, se cambia el orden institucional, haciendo depender las misiones, objetivos y tareas de la organización ya definida. Así, un estatuto moderno se puede entender, para nuestro específico caso, como un cuerpo de ordenación superior que abordara los aspectos de la misión institucional, las relaciones con el Estado, el gobierno superior y los deberes y derechos de sus miembros. Lo anterior permite disponer de un cuerpo estatutario superior flexible, acotado a los aspectos fundamentales, y que permite focalizar cuestiones operativas, como estructura organizativa, organización de los estudios, mecanismos de generación de autoridades y otros, en reglamentos de diferente rango.

Así se abre un cauce amplio para determinar el tipo de gobierno superior, con seguridad en la figura de un Rectory autoridades ejecutivas junto a un órgano normativo que establezca, más allá de las estructuras, las grandes orientaciones estratégicas de la Universidad. El gobierno superior deberá estar en manos de un poder ejecutivo, trabajando en estrecha relación con un órgano normativo independiente y autónomo en su misión de entregar las líneas orientadoras del desarrollo universitario.

De este modo caminaremos hacia formas de gobierno superior solidario con la Universidad, con visión de conjunto de la temática nacional e internacional, con estabilidad y consistencia frente a metas de largo plazo y con capacidad de respuesta frente a una sociedad demandante no sólo de servicios, sino también de valores.

Otro aspecto de difícil tratamiento por lo inmediato de las urgencias que se establecen a su alrededor, es el de las formas de gestión. Nuestra Universidad mantuvo hasta finales de la década del '80 las mismas formas de gestión que venían desarrollándose veinte años antes. La intervención militar en la Universidad c a lv o muy pequeñas modificaciones - dio vuelta la espalda a los rápidos cambios que ocurrían en el exterior, no entendiendo que la gestión universitaria en las instituciones estatales era cada vez más dependiente de factores ligados al medio ambiente circundante. Nuestras Universidades se hacían cada vez más independientes del 
Estado no sólo por la caída del financiamiento, sino también por la dinámica, aunque lenta, de poner al día las tradiciones y maneras de realizar sus trabajos y tareas. Poner en tensión la tradición y cultura universitaria es un riesgo evidente en el esfuerzo de indagar nuevas formas de gestión. "Pasar de los actuales mecanismos de planificación universitaria a un ambiente de planificación estratégica, significa un cambio sustantivoen el modo de gestión para el cual, posiblemente, las Universidades y sus académicos no estén preparados. Razonar en términos de las oportunidades y amenazas del medio externo, realizar un análisis sistemático y acabado de las fortalezas y debilidades internas, barajar escenarios alternativos y generar, en definitiva, planes estratégicos, no son cuestiones que los académicos, ni siquiera los directivos universitarios, tengan el hábito de hacer y que, posiblemente, encuentren en un primer momento severas resistencias" 5 .

Por lo tanto, considerar que la dotación universitaria debe ser visualizada como una variable de gestión es, aun hoy díí, una cuestión del todo ajena a la vida universitariay, en algunos casos, atentatoria del quehacer académico.

Por cta parte, la Universidad recibe fuertes críticas por el gasto en remuneraciones que mantiene en la actualidad, sin que ellas se detengan por un instante en una perspectiva de más largo plazo, teniendo a la vista la situación de ingresos del pasado.

Ambas urgencias no permiten considerar, con la debida atención, el tema relevante, nuevamente casi utópico, cual es que el recurso humano debe ser entendido como la herramienta primordial de desarrollo y cambio, para lo cual se requiere disponer de mecanismos de selección, promoción, reconocimiento y egreso que ayuden a captar a los mejores estudiantes y a los más capacitados y distinguidos profesores.

Afortunadamente, las nuevas experiencias y tecnologías para una moderna gestión académica nos permiten ser extraordinariamente optimistas frente a eventuales cambios.

Estoy convencido de que, en la medida en que podamos romper esta disyuntiva paralizadora y abordar sinérgicamente lo urgente y lo trascendente, en otras palabras, de transitar con fluidez de la coyuntura a la utopía, habremos dado nuevamente un paso importante al interior de nuestra Universidad y, por cierto, en el sistema de Educación Superior.

5Basso, Patricio, "Grandesdesafios del sistema universitarioen la última década del siglo xx", en Pablo Pérsico (editor), Educación Superior chilena: Gestión y administración institucional, III Foro de la Educación Superior,CPU, 1992. 
Tengo la más plena confianza que así lograremoscumplir más pronto la misión institucional: Contribuin a la formación del bombre y de la mujer chilenos y al desarrollo espiritual, cultural y material del pais; aportar a la solución de los grandes problemas nacionales, velar por el bien común, preservar ei medio ambiente, enriquecer el patrimonio cultural de Chiley su identidad, y contribuir aldesarralloy mejoramiento del sistema universitario nacional. 